\title{
The intensional side of algebraic-topological representation theorems
}

\author{
Negri, Sara
}

2017

Negri , S 2017 , ' The intensional side of algebraic-topological representation theorems ' , Synthese , vol. 198 , no. Suppl 5 , pp. 1121-1143 . https://doi.org/10.1007/s11229-017-1331-1

http://hdl.handle.net/10138/307561

https://doi.org/10.1007/s11229-017-1331-1

Downloaded from Helda, University of Helsinki institutional repository.

This is an electronic reprint of the original article.

This reprint may differ from the original in pagination and typographic detail.

Please cite the original version. 


\title{
The intensional side of algebraic-topological representation theorems
}

\author{
Sara Negri \\ Department of Philosophy, History, Culture and Art Studies, \\ P.O. Box 24, FIN-00014, University of Helsinki \\ FINLAND \\ sara.negri@helsinki.fi
}

\begin{abstract}
Stone representation theorems are a central ingredient in the metatheory of philosophical logics and are used to establish modal embedding results in a general but indirect and non-constructive way. Their use in logical embeddings will be reviewed and it will be shown how they can be circumvented in favour of direct and constructive arguments through the methods of analytic proof theory, and how the intensional part of the representation results can be recovered from the syntactic proof of those embeddings. Analytic methods will also be used to establish the embedding of subintuitionistic logics into the corresponding modal logics. Finally, proof-theoretic embeddings will be interpreted as a reduction of classes of word problems.
\end{abstract}

\section{Introduction}

Algebraic-topological representation theorems have a long history, dating back to the work of Marshall Stone on the representation of Boolean algebras (1936), with the spaces that arise from such representation bearing his name. As emphasized in the historical introduction of Johnstone (1982), the legacy of Stone representation theorems has affected almost all fields of mathematics, having been a tool not only in logic but also in topology, with the study of its fundamental structures from a lattice-theoretic and categorical viewpoint (locale theory and topos theory), and encompassing also the areas of algebraic geometry and functional analysis.

The common aspect of such theorems is the representation of algebraic structures in terms of set-theoretic ones, often endowed with a topology. Ordered 
algebraic structures are an abstraction of the properties of Boolean operations of intersection, union, and complementation in sets, with the order corresponding to set inclusion, and can be considered as an intensional way of conceptualizing the extensional properties of topological spaces.

The shift from intensional to extensional definitions is a delicate one, and in fact, the move requires the use of a non-constructive principle to assert the existence of ideal elements. The same shift is encountered when topological notions are formalised intensionally in approaches such as formal or pointfree topology ${ }^{1}$, specifically in proving that these new topologies can be made equivalent to the traditional, point-set ones.

The wide area of algebraic logic has its foundations in duality theory that in turn depends largely on algebraic-topological representations. In particular, Stone representation theorems are a central ingredient in the metatheory of philosophical logics. In this field, one of the most well known uses of Stonetype representations is in the embedding of intuitionistic logic into modal logic, achieved, through completeness, with the representation of complete Heyting algebras as closure spaces. ${ }^{2}$

The representation of Heyting algebras in terms of closure algebras is used inand in a way corresponds to - the proof of faithfulness of the translation of intuitionistic logic, Int, into the modal logic S4. In turn, the embedding of intuitionistic logic into the modal logic $\mathbf{S} \mathbf{4}$ was obtained as an attempt to give a provability interpretation of intuitionistic logic. Gödel's (1933) sound translation from Int into $\mathbf{S} 4$ was proved faithful as long as 15 years later by McKinsey and Tarski (1948). The result was then generalised by Dummett and Lemmon (1959) who extended it to intermediate logics between Int and classical logic and the corresponding intemerdiate logics between S4 and S5. Like the result by McKinsey and Tarski, Dummett and Lemmon's proof used the same indirect semantic method, namely the completeness of intuitionistic logic with respect to Heyting algebras (there called Brouwerian algebras) and of $\mathbf{S 4}$ with respect to topological Boolean algebras (called closure algebras). The algebraic part of the proof consists in the representation of Heyting algebras as the opens of topological Boolean algebras. The proof is therefore indirect because it is obtained by algebraic means through completeness, and non-constructive because of the use of Stone representation of distributive lattices, in particular Zorn's lemma. In more recent literature, surveyed by Wolter and Zakharyaschev (2014), embedding results are established semantically and by use non-constructive principles.

All these results obtained by semantic means establish correspondences with respect to derivability between superintuitionistic systems and normal extensions of S4. In particular, they do not show how to translate a proof from one system to the other. The interest in obtaining a direct proof of the faithfulness result was therefore motivated not only by the need of adhering to a strict purity of

\footnotetext{
${ }^{1}$ Cf. Sambin (1987).

${ }^{2}$ Heyting algebras are also called pseudo-Boolean algebras, e.g. in Rasiowa and Sikorski (1963).
} 
methods.

It has been shown (Dyckhoff and Negri 2012) that logical embeddings can be obtained as translations of derivations, in a direct way that does not employ the non-constructive principles typical of the representation theorems. The method is based on the uniform development of analytic proof systems for the logics in question. Analytic proof systems stem from the tradition of Gentzen systems of sequent calculi, with the characteristic feature that in a proof only syntactic objects ranging in a set which is predetermined by the statement to be proved are used. If one uses axiomatic systems for embedding results, the soundness part of the proof can be given, as Gödel did, by induction on derivations. For the faithfulness part, however, this is not in general possible. The reason is that when proceeding inductively one needs to have in the derivations premisses to which the inductive hypothesis can be applied, but the inductive hypothesis can be applied only to formulas in the range of the translation. Without a calculus with sufficient analyticity properties, in particular with an axiomatic system, the premisses of the last rule used in the derivation can be very far from a formula of that form, and the inductive hypothesis cannot in general be applied. Sequent calculi with cut elimination and the subformula property are a remedy to this shortcoming of axiomatic systems and thus give the potentially appropriate tool for establishing results of this kind directly.

When such systems can be developed in a uniform way for a family of logics, through the definition of a common core calculus to which further rules are added, they can be used not just for studying the relation of logical consequence of a given logic, but for establishing metatheoretic results, such as embeddings, on the relationship between different logics. Analyticity and uniformity are the key features that reduce the proof of faithfulness of those embeddings to an induction on derivations, and thus one avoids the need of the detour through completeness and Stone-representations. Analyticity gives full control on the structure of derivations in the frame classes considered. Uniformity, on the other hand, guarantees that the same procedure works in all the corresponding systems because it operates only on the common core calculi and leaves unchanged the added rules. As a further evidence of its generality and flexibility, we show how the method can be employed to establish also the embedding of subintuitionistic logics into the corresponding modal logics below $\mathbf{S} 4$.

The general picture that emerges with the development of uniform analytic methods for non-classical logics not only shows that the indirect and nonconstructive proofs based on Stone representation can be avoided in the proof of such embeddings, but also suggests a change in perspective, that is, to privilege the use of logical methods, and more specifically, proof-theoretic methods, to recover the intensional, pointfree part of representation results. By the identification of free Heyting algebras with the Lindenbaum algebras of intuitionistic logic, the logical embedding gives an embedding of freely generated Heyting algebras in closure algebras. Finally, thanks to the precise sense in which the inductive generation of syntactic objects in a sequent calculus parallels the inductive generation of equalities between terms in a free algebra, the 
proof-theoretic embedding can then be interpreted as a reduction of classes of word problems.

The paper is organized as follows: Section 2 gives background notions and a summary of the current literature and results on the algebraic-topological representation theorems and their use in logical embeddings. Some features concerning modal embedding results obtained by traditional (semantic) means are analyzed: in particular, the proof of such results uses indirect and nonconstructive proofs based on Stone-type representation theorems. Section 3 presents two main lines of enquiry directed at the isolation and avoidance of non-constructive principles in Stone-like theorems and their applications. The first is given by the development of constructive mathematics within formal topology, discussed in Section 3.1, the second by analytic proof theory, especially labelled sequent calculi. Section 3.2 shows how uniform analytic methods for non-classical logic can be employed to establish a proof-theoretic embedding of intermediate and subintuitionistic logic into the corresponding modal logic. Finally, Section 4 addresses how to interpret proof-theoretical embeddings as a reduction of classes of word problems. The conclusion summarizes the main point of this paper and contains pointers to literature that discusses Stone representation theorems from a philosophical perspective.

\section{Algebraic-topological representation theorems}

Stone-type representation theorems characterize abstract ordered and algebraic structures in terms of suitable fields of sets or topological spaces.

The most well known such theorem is the Stone representation theorem for Boolean algebras (1936). On the one hand it is immediate to verify that the set of subsets of a set constitutes a Boolean algebra with the operations of intersection, union, and complement, on the other it is not obvious that fields of sets, i.e. families of sets closed under such operations, are sufficiently general to be put into correspondence with Boolean algebras. The representation theorem solves the problem of showing that any abstract Boolean algebra is isomorphic to a field of sets, indeed an appropriate subspace thereof.

Another celebrated representation result is the topological representation theorem for complete Heyting algebras which states that the opens of a topological space form a complete Heyting algebra and conversely that every complete Heyting algebra with a suitable additional property can be obtained in this way: For every complete Heyting algebra with enough points ${ }^{3} H$, there is a topological space $X$ such that

$$
H \cong \mathcal{O}(X)
$$

3 The condition of having enough points, or being spatial, means that if $a \nless b$ in the algebra, there is a formal point that contains $a$ but not $b$, that is, the (formal) points are enough to witness the order relation in the algebra. See Johnstone (1982) for the condition of spatiality in locale theory and Gambino and Schuster (2007) for a survey on spatiality in formal topology. 
where $\mathcal{O}(X)$ is the ordered algebra of open sets of $X$.

Instead of recalling the basic ideas of the afore-mentioned representation, we start from the result that presupposes the minimum of structure and then indicate how the representation is enriched.

To make the presentation self-contained, we shall also briefly recall in the notes the order-theoretic notions used; however, for a comprehensive background the reader can consult Davey and Priestley (1990).

Given a partially ordered set $^{4}(P, \leq)$ we can associate to it in a natural way a partially ordered set of sets. We say that a subset $C$ of $P$ is a cone if it is upward closed, that is, for all $x, y$ in $P$, if $x \in C$ and $x \leq y$, then $y \in C$. Then, to any element $a$ of $P$, we associate the set of cones included in $P$ that contain $a$, denoted by $h(a)$. It is then easy to show that $a \leq b$ if and only if $h(a) \subseteq h(b)$ : If $a \leq b$ and $C$ is a cone in $h(a)$, then by definition of cone and the assumption we have that $b \in C$, thus $C$ is in $h(b)$ as well. For the converse, $\uparrow a$, defined as the set $\{x \in P: a \leq x\}$, is a cone that contains $a$ (called the principal cone generated by $a$ ) and the assumption gives $b \in \uparrow a$, which is the same as $a \leq b$. It is equally easy to see, just using antisymmetry of $\leq$, that the map $h$ is injective, thus giving an embedding of $(P, \leq)$ into $(\mathcal{P}(\mathcal{P}(P)), \subseteq)$, the double powerset of $P$ ordered by set-theoretic inclusion.

In a similar way one can represent a semilattice, ${ }^{5}(L, \wedge)$, and the operation that corresponds to $\wedge$ on the set-theoretic side is intersection. In this case as a map $h$ one takes $h(a) \equiv \downarrow a$, where $\downarrow a \equiv\{x \in P: x \leq a\}$, called the dual principal cone generated by $a$. The map is a semilattice homomorphism from $(L, \wedge)$ to $(\mathcal{P}(P), \cap)$ since $h(a \wedge b)=h(a) \cap h(b)$ immediately follows from the $\wedge$ semilattice laws; it is also injective and therefore an embedding of semilattices. The non-triviality of representation results is seen already when by the same method one attempts to represent lattices other than semilattices. One would expect that the set-theoretic operation that corresponds to $\vee$ would be the union, however, sets with union and intersection always form a distributive lattice, ${ }^{6}$ whereas lattices are not, in general, distributive. It is unproblematic to represent semilattices with either meet or join, but when both operations are involved it is not possible to obtain the representation in an easy way from the representations of both semilattice structures.

Leaving the problem of representation of arbitrary lattices aside, we turn to

\footnotetext{
${ }^{4}$ We recall that a relation $\leq$ on a set $P$ is a partial order if it is reflexive, transitive, and antisymmetric, i.e. if it satisfies $\forall x . x \leq x, \forall x y z . x \leq y \& y \leq z \rightarrow x \leq z$, and $\forall x y . x \leq y \& y \leq$ $x \rightarrow x=y$. Since a partial order is an antisymmetric preorder and antisymmetry holds by definition if equality is defined by the $\leq$ relation in the two direction, preorders are often preferred to partial orders as more basic structures.

${ }^{5}$ We recall that a partially ordered set $L$ is a meet-semilattice if for any two elements $a, b$ of $L$ the greatest lower bound of $a$ and $b$ exists, i.e. there is a binary operation $\wedge$ on elements of $L$ with the properties $a \wedge b \leq a, a \wedge b \leq b$, and $\forall c . c \leq a \& c \leq b \rightarrow c \leq a \wedge b$.

${ }^{6} \mathrm{~A}$ lattice is distributive if the operations of meet and join distribute over each other, i.e. the condition $a \wedge(b \vee c)=(a \wedge b) \vee(a \wedge c)$ holds for arbitrary elements of the lattice. By a general result in lattice theory, the condition is equivalent to the dual condition $a \vee(b \wedge c)=$ $(a \vee b) \wedge(a \vee c)$.
} 
distributive lattices. Also in this case, the map $h$ defined by $h(a) \equiv\{x \in L$ : $x \leq a\}$ fails to be a homomorphism between $L$ and $\mathcal{P}(L)$. An example can be constructed of a lattice where $a \vee b$ is the top element but $h(a) \cup h(b)$ is not. ${ }^{7}$ The requirement for this to happen is that the map $h$ ranges among elements of $\mathcal{P}(L)$ with the property that $x \leq a \vee b$ implies $x \leq a$ or $x \leq b$, i.e. satisfy the condition of being prime. A filter is a non-empty set which is upward closed and closed under meets. A prime filter $P$ thus satisfies the following closure properties

$$
\begin{gathered}
a \wedge b \in P \text { if and only if } a \in P \text { and } b \in P \\
a \vee b \in P \text { if and only if } a \in P \text { or } b \in P
\end{gathered}
$$

These imply immediately that the map

$$
\begin{aligned}
h: L & \rightarrow \mathcal{P}(L) \\
a & \mapsto h(a) \equiv\{P \in \mathcal{P}(L): P \text { prime filter, } a \in P\}
\end{aligned}
$$

is a homomorphism from $(L, \wedge, \vee)$ to $(\mathcal{P}(P), \cap, \cup)$. Injectivity of the map instead calls into play the following separation principle, known as the prime filter theorem:

Lemma 2.1. Let $L$ be a distributive lattice, and $a, b$ two elements of $L$ with $a \not \leq b$. Then there exists a prime filter $P$ such that $a \in P$ and $b \notin P$.

The proof of this result (cf. e.g. Dunn and Hardegree 2001 for details) consists in considering the set $E$ of filters that contain $a$ but not $b$. Since by assumption $a \not \leq b$, the principal filter $\uparrow a$ is in $E$ which is thus nonempty. Then, given an arbitrary chain $C$ in $E$ it is easy to verify that the union of $C$ is also in $E$. By Zorn's lemma, $E$ has a maximal element, call it $P$. To show that $P$ is prime, we reason by contradiction and assume that there are $c, d$ with $c \vee d$ in $P$ but neither $c$ nor $d$ in $P$. Let $(P, e)$ be the filter $\{y: x \wedge e \leq y$ for some $x \in P\}$. Then the filters $F_{1} \equiv(P, c)$ and $F_{2} \equiv(P, d)$ are proper extensions of $P$ and therefore cannot be in $E$, so they have to contain $b$. It follows that we have $x \wedge c \leq b$ and $y \wedge d \leq b$ for some $x, y$ in $P$, and therefore, taking $z=x \wedge y$ we have $z \wedge c \leq b$ and $z \wedge d \leq b$, and thus $(z \wedge c) \vee(z \wedge d) \leq b$, so using the lattice laws and distributivity we have $z \wedge(c \vee d) \leq b$. But then $b$ would be in $P$, against the fact that $P$ is a filter in $E$.

The topological part of Stone representation theorem for distributive lattices (1937, theorem 15) follows from defining a topology in the space of prime filters over $L$ taking as a base the sets $h(a)$. In the corresponding point of the construction, Stone takes ideals rather than filters, and denotes the set of ideals that contain $a$ with $\mathfrak{S}(\mathfrak{a})$; we observe that these are dual notions so the difference is inessential. The sets $h(a)$ are then seen to characterize the compact elements of the topology. A topological space generated by a base of compact sets and where the compact sets form a meet semilattice is called a coherent space. The representation theorem establishes the following:

\footnotetext{
${ }^{7}$ Cf. Dunn and Hardegree (2001), p. 293.
} 
Theorem 2.2 (Stone representation theorem for distributive lattices). Every distributive lattice is isomorphic to the lattice of compact elements of a coherent space.

The representation theorem for distributive lattices can then be specialised, by using the same map, to a representation theorem for complete Heyting algebras ${ }^{8}$ with enough points as the algebras of opens and complete Boolean algebras as the algebras of clopens (sets which are both open and closed) of suitable topological spaces. For our purposes, we needn't go into the details of these results that can be found for example in Johnstone (1982). We also just mention here that the duality theory for general Heyting algebras, rather than just complete Heyting algebras, has followed a line of development started with Priestley duality of bounded distributive lattices (Priesley 1970, see also ch. 10 of Davey and Priestley 1990), the special case of Esakia's duality for Heyting algebras, and a bitopological duality in the work by Bezhanishvili et al. (2010). ${ }^{9}$ We observe that the common ingredient of these representations of ordered-algebraic structures as lattices/algebras/fields of sets is the embedding of the orderedalgebraic structure into its corresponding algebra of prime filters, that play the role of points of the topological space. On the one hand, as we have seen above, proving that the map that gives the embedding is injective requires the use of the non-constructive separation principles of the prime filter theorem, on the other, the topological part of the representation is just a plain application of the definitions involved. Taking an inductively defined notion of cover relation as a primitive, rather than the notion of point, should therefore allow to isolate the constructive content of the representation, and in fact this is what is achieved through the formal approach to topology which has been developed in two main lines of investigation, that of locale theory and category theory, and that of formal topology (we shall return to this in Section 3.1 below).

\subsection{Embedding of Heyting algebras into closure algebras}

Instead of representing Heyting algebras externally through the space of open sets of a topological space, one can abstract from the set-theoretic notion of openness and consider, rather than a topological space, the abstract algebraic structure of a Boolean algebra endowed with an operation that has the same

\footnotetext{
${ }^{8}$ We recall that an Heyting algebra is a distributive lattice endowed with an operation $\rightarrow$ that satisfies $a \wedge b \leq c$ if and only if $a \leq b \rightarrow c$. A complete Heyting algebra is an Heyting algebra which is complete as a lattice, i.e. one in which the supremum (least upper bound) and infimum (greatest lower bound) exist for arbitrary subsets of elements. By a known result (cf. e.g. I.4.3 of Johnstone (1982) it is enough to require closure under arbitrary meets (resp. joins) to obtain closure under arbitrary joins (resp. meets), i.e. a complete semilattice is also a complete lattice. The two notions are however distinct when morphisms are considered because a map that preserves arbitrary joins (resp. meets) need not preserve arbitrary meets (resp. joins).

${ }^{9}$ This latter paper gives a useful summary of the results in duality theory in this line of investigation.
} 
properties as those of a closure operator. The representation can thus be formulated internally, without mentioning spaces of points. We recall some definitions.

Definition 2.3. $A$ closure algebra $(B, c)$ is a Boolean algebra $B$ endowed with $a$ closure operator $c$, i.e. an operator that satisfies the following properties:

$$
\begin{aligned}
& \text { 1. } c 0 \leq 0 \\
& \text { 2. } c(x \vee y)=c x \vee c y \\
& \text { 3. } x \leq c x \\
& \text { 4. } c c x \leq c x
\end{aligned}
$$

Definition 2.4. Given a closure algebra $(B, c)$, an interior operator is defined as the dual of a closure operation by $i a \equiv-c-a$. The open elements of $(B, c)$ are the elements $a$ of $B$ such that $a=i a$.

We recall that given a topological space $(X, \mathcal{O}(X))$, a complete Heyting algebra $H$ is obtained by taking as elements the open sets $\mathcal{O}(X)$ of the topological space, as lattice operations union and intersection, and as implication of two open sets $a$ and $b$ the topological interior of the union of $b$ and the complement of $a$, $\mathcal{I}((X-a) \cup b)$. In an analogous way, given a closure algebra $(B, c)$, a Heyting algebra $H$ can be defined as the abstract version of the Heyting algebra of open sets of a topological space. The algebra is obtained by taking as elements the open elements of $B$, as lattice operations $\wedge, \vee$ the lattice operations of $B$, and as implication

$$
a \rightarrow b \equiv i(-a \vee b)
$$

Conversely, every Heyting algebra can be obtained as the algebra of open elements of a closure algebra. The result is proved by using the prime filter theorem and is therefore not constructive.

\section{$3 \quad$ Avoiding non-constructive principles}

As indicated in the previous sections, algebraic-topological representation theorems depend essentially on non-constructive principles in the construction of ideal elements that connect their intensional and extensional sides. We shall outline two ways in which such non-constructive principles are first isolated, and then avoided. The first is the use of formal, rather than point-set, topology. The second is the use of the methods of analytic proof theory.

\subsection{Pointfree topology}

Stone representations and their applications were studied systematically by Johnstone (1982) through the use of the unifying conceptual framework of category theory and, more specifically, locale theory. The avoidance of the use of 
non-constructive principles was, however, the main emphasis in another strand of developments of topology in a synthetic fashion known as formal (pointfree) topology (Sambin, 1987). Predicativity is guaranteed in formal topology by a formalization of its basic notions and results within Martin-Löf's constructive type theory (Martin-Löf, 1984). Other developments that started from Stone representation and more specifically directed to the semantic study of modal logic include the work on duality theory initiated by Leo Esakia and brought further by his many followers, as is well documented in a recent volume (Bezhanishvili, 2014).

In formal topology, the order-theoretic properties of a topological space are assumed as primitive by means of a relation, called formal cover, between elements $a$ and subsets $U$ of a set $S$. The properties that a cover relation $a \triangleleft U$ has to satisfy correspond to the properties of the set-theoretic relation of inclusion $a \subseteq \bigcup U$ between basic neighbourhoods and the union of a set of basic neighbourhoods of a topological space. Formal topology is pointfree, as the points are not primitive objects, and constructive, because its underlying set theory is. Points are not among the primitive notions of formal topology, but are defined as particular, well-behaved collections of neighbourhoods (in lattice-theoretic terms, completely prime filters over the basic opens of the formal topology). Formal spaces are the spaces that arise as spaces of formal points of a suitable formal topology.

As we already hinted at in discussing the representation of distributive lattices, the constructive content of Stone representation can be made evident by suitably restricting the representation to the pointfree part of the representing spaces.

The representation theorem for complete Heyting algebra (equivalently called frames, i.e. complete lattices with finite meets that distribute over arbitrary joins) takes the form (see Sambin 1987):

Every frame $F$ is isomorphic to the frame of saturated subsets and to the frame of formal opens of a formal topology $\mathcal{A}$

$$
F \cong \operatorname{Sat}(\mathcal{A}) \cong \operatorname{Open}(\mathcal{A}) \equiv \mathcal{P}(\mathcal{S}) /=_{\mathcal{A}}
$$

Here $\operatorname{Sat}(\mathcal{A})$ denotes the collection of subsets $U$ of the base $S$ of the formal topology which are closed with respect to the closure operator induced by the formal topology $\mathcal{A}$, i.e., which satisfy $\mathcal{A} U=U$, where $\mathcal{A} U$ consists of the $a$ in $S$ such that $a \triangleleft U$. The frame of formal opens, $\operatorname{Open}(\mathcal{A})$ is the quotient of $\mathcal{P}(S)$ modulo the equivalence relation

$$
U={ }_{\mathcal{A}} V \equiv U \triangleleft V \& V \triangleleft U .
$$

The isomorphism is established by the assignment that selects, for each equivalence class $[U]$, the canonical representative $\mathcal{A} U$ using the fact that $U={ }_{\mathcal{A}} V$ if and only if $\mathcal{A} U=\mathcal{A} V$.

Several extensions of constructive Stone representation based on formal spaces were given in Negri $(1996,2002)$. They cover distributive lattices and lattice 
structures used in abstract computable analysis and domain theory, such as continuous, prime-continuous, algebraic lattices, continuous and algebraic dcpo's, Scott domains; they do not use any non-constructive principles, which might at first sight seem surprising since Stone representation relies in its very heart on Zorn's lemma. Indeed, one would be more cautious in saying that pointfree topology allows to isolate, rather than avoid, non-constructive principles; let us see why. We recall the way in which pointfree spaces are put into correspondence with standard topological spaces, that is, how pointfree topology is matched with point-set topology (in addition to the aforementioned literature, for a compact introduction to the basic notions of formal topology we refer to section 2 of Negri, 2002).

Topological spaces can be turned into formal spaces by considering the formal points over any base for their topology

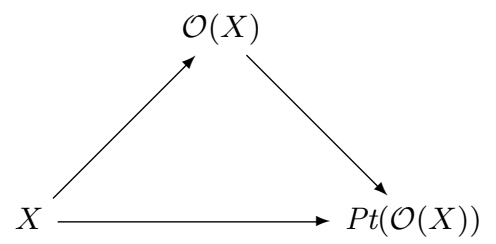

We have $X \cong \operatorname{Pt}(\mathcal{O}(X))$ if the space $X$ satisfies a weak separation principle called sobriety. The principle states that any irreducible closed set is the closure of a unique point. To locate the principle in the hierarchy of separation principles in topology it suffices to say that the principle is incomparable with $T_{1}$ (for any two distinct points there is a neighbourhood that contains one but not the other) but weaker than $T_{2}$ (any two distinct points are separated by disjoint neighbourhoods). Sobriety is generically expressed by the slogan there are enough opens.

Conversely, formal topologies are turned into point-set spaces as follows: sets of formal points are endowed with the extensional topology which takes as basic opens the set of formal points that contain a basic open $a$, denoted by $\operatorname{ext}(a)$. This is the counterpart of the set of prime filters $h(a)$ in Stone representation.

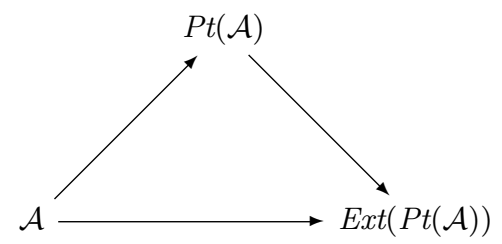

We have $\mathcal{A} \cong \operatorname{Ext}(\operatorname{Pt}(\mathcal{A}))$ if the formal topology $\mathcal{A}$ is spatial, or extensional or, in a slogan, has enough points. The property is

$$
\operatorname{ext}(a) \subseteq \operatorname{ext}(U) \text { implies } a \triangleleft U
$$

Observe that this is an implication that goes from the extensional inclusion between two sets of formal points to a formal cover relation, which can be 
presented in many interesting cases as an inductively defined relation, ${ }^{10}$ and therefore moves from a universal property to an existential one, exactly as in a completeness theorem. Indeed, the property of extensionality for formal spaces can be made to correspond to completeness in a very precise sense which was made clear already by Fourman and Grayson (1982). As for completeness of a logical system, also extensionality cannot in general be established constructively. For example, proving extensionality for locally Stone formal topologies requires Zorn's lemma (cf. Negri, 2002).

In this sense, one may regard formal spaces as theoretical entities that allow the separation of two components: the first, based on formal basic opens and formal covers, is often used to reformulate and prove constructively theorems that in their traditional formulation belong to classical topology and analysis; the second component, based on formal points, puts formal spaces in correspondence with traditional, point-set, spaces. One finds the use of non-constructive principles in the proofs of equivalence of the formal and point-set part, so what one achieves is the isolation, rather than complete avoidance, of non-constructive principles of proof. In the next section we shall show how analytic proof theory goes further in the avoidance of non-construcive principles.

\subsection{Analytic proof theory}

The representation of Heyting algebras in terms of closure algebras is used in, and in a way corresponds to, the proof of faithfulness of the translation of intuitionistic logic, Int, into the modal logic $\mathbf{S} 4$. We are going to recall from Dyckhoff and Negri (2012) how analytic proof systems allow a direct and constructive proof of the result that was previously established by algebraic means. Further, we shall show that not only the non-constructive reasoning of algebra can be avoided by these means, but that the algebraic result itself can be recovered through the methods of proof theory.

We recall that the embedding of intuitionistic logic into the modal logic $\mathbf{S} \mathbf{4}$ was obtained as an attempt to give a provability interpretation of intuitionistic logic (see, however, the introduction of Dyckhoff and Negri 2016 for a discussion on the suitability of $\mathbf{S} \mathbf{4}$ for such an interpretation). In his note of 1933, Gödel defined a translation from Int into $\mathbf{S} 4$ and proved its soundsness, i.e. that

$$
\vdash_{\text {Int }} A \rightarrow \vdash_{S 4} A^{*}
$$

\footnotetext{
${ }^{10}$ Inductive generation of formal covers has been the key property in the presentation of formal reals, formal intervals and formal linear functionals for establishing results such as the constructive version of the Tychonoff, the Heine-Borel, and the Hahn-Banach theorem and the representation of continuous domains (cf. Negri and Soravia, 1999; Negri and Valentini, 1997; Cederquist and Negri, 1996; Cederquist, Coquand, and Negri, 1998; Negri, 2002). For a survey on inductive generation of formal topologies in the wider context of inductive definitions in type theory and examples of formal topologies that cannot be inductively generated cf. Coquand, Sambin, Smith, and Valentini (2003). For examples of inductively generated formal topologies see also section 4 of Gambino and Schuster (2007).
} 
and only conjectured faithfulness, i,e, that also the converse

$$
\vdash_{S 4} A^{*} \rightarrow \vdash_{\text {Int }} A
$$

holds. It took 15 years before the conjecture was proved true in McKinsey and Tarski (1948), who showed semantically the contrapositive of faithfulness, i.e. that

$$
\nvdash_{\text {Int }} A \rightarrow \nvdash_{S 4} A^{*}
$$

The result was then generalised by Dummett and Lemmon (1959) who extended it to intermediate logics between Int and classical logic, obtained by the addition of an arbitrary propositional axiom $A x$ to intuitionistic logic and to the corresponding intermediate logics between $\mathbf{S} \mathbf{4}$ and $\mathbf{S 5}$, the latter obtained by adding the modal translation of the same axiom

$$
\vdash_{\text {Int }+A x} A \text { if and only if } \vdash_{S 4+A x^{*}} A^{*}
$$

The latter result was a generalisation of the former of McKinsey and Tarski and used the same indirect semantic method, namely the completeness of intuitionistic logic with respect to Heyting algebras (there called Brouwerian algebras) and of $\mathbf{S} \mathbf{4}$ with respect to topological Boolean algebras, also called closure algebras, which are Boolean algebras endowed with an operator axiomatized through the properties of a topological operator of interior. The algebraic part of the proof consists in the representation of Heyting algebras as the opens of topological Boolean algebras. The proof is therefore indirect because it is obtained by algebraic means through completeness and non-constructive because of the use of Stone representation of distributive lattices, in particular Zorn's lemma.

A survey of the classical embedding results and of more recent related literature can be found in section 9.6 of the monograph by Chagrov and Zakharyaschev (1997). We just recall that one can define various maps from intermediate (also called superintuitionistic or s.i.) logics (ExtInt) and normal extensions of S4 (NExtS4). For the canonical translation $T$, given $L \equiv$ Int $+\Gamma$, there is, by the result of Dummett and Lemmon restated in other words in the above, an associated modal logic

$$
\tau \mathrm{L} \equiv \mathbf{S} \mathbf{4} \oplus\{T(A) \mid A \in \Gamma\}
$$

such that for every $A \in \mathcal{L}, A \in \mathrm{L}$ iff $T(A) \in \tau \mathrm{L}$. One can define two more maps $\rho:$ NExtS4 $\rightarrow$ ExtInt and $\sigma:$ ExtInt $\rightarrow$ NExtS4 by $\rho \mathrm{M} \equiv\{A \in \mathcal{L} \mid T(A) \in \mathrm{M}\}$ and $\sigma \mathrm{L} \equiv \tau \mathrm{L} \oplus \mathbf{G r z}$, where $\mathbf{G r z}$ is the extension of $\mathbf{S} 4$ with axiom $\square(\square(A \supset$ $\square A) \supset A) \supset A$. The logic $\rho \mathrm{M}$ was called by Esakia the superintuitionistic fragment of $\mathrm{M}$ and $\mathrm{M}$ the modal companion of $\mathrm{L}$. For an intermediate logic there is no unique modal companion, and indeed there is a smallest and largest modal companion. For Int the smallest is S4 and the largest Grz. Various results have been established on the relations between the lattice of superintionistic logics and the lattice of normal extensions of $\mathbf{S 4}$, and we recall in particular the 
Blok-Esakia theorem by which the map $\rho$ is a lattice isomorphish from ExtInt onto NExtGrz. ${ }^{11}$

All these results are proved by semantic means and establish correspondences with respect to derivability between superintuitionistic systems and normal extensions of $S 4$. In particular they do not show how to translate a proof in the companion of $L$ to a proof in $L$. The interest in obtaining a direct proof of the faithfulness result was therefore motivated not only by the need of adhering to a strict purity of methods. The difficulty in establishing the result using only the methods of proof theory is the following: for the proof of soundness one can use the well established axiomatic system that allows to define derivations inductively. The proof can thus be given, as already Gödel did, by induction on derivations. For the faithfulness part, however, this is not in general possible. The reason is that when proceeding inductively one needs to have in the derivations premisses to which the inductive hypothesis can be applied, but the inductive hypothesis can be applied only to formulas in the range of the translation. Without a calculus with sufficient analyticity properties, in particular with an axiomatic system, the premisses of the last rule used in the derivation can be very far from a formula of that form, and the inductive hypothesis cannot in general be applied. Sequent calculi with cut elimination and the subformula property remedy this shortcoming of axiomatic systems and thus give the potentially appropriate tool for establishing these kinds of results directly. The first direct proof of faithfulness of the modal embedding of intuitionistic logic was in fact given in Troelstra and Schwichtenberg (1996) through a cut-free sequent calculus for $\mathbf{S} 4$. The following variant of the original Gödel translation was used:

$$
\begin{aligned}
P^{\square} & :=\square P \\
\perp^{\square} & :=\perp \\
(A \supset B)^{\square} & :=\square\left(A^{\square} \supset B^{\square}\right) \\
(A \& B)^{\square} & :=A^{\square} \& B^{\square} \\
(A \vee B)^{\square} & :=A^{\square} \vee B^{\square}
\end{aligned}
$$

The translation $\Gamma^{\square}$ of a multiset $\Gamma \equiv A_{1}, \ldots, A_{n}$ is defined componentwise by

$$
\left(A_{1}, \ldots, A_{n}\right)^{\square}:=A_{1}^{\square}, \ldots, A_{n}^{\square}
$$

Soundness and faithfulness for the translation of the relation of logical consequence in sequent calculus is formulated as

$$
\text { Int } \vdash \Gamma \rightarrow A \text { if and only if } \mathbf{S} 4 \vdash \Gamma^{\square} \rightarrow A^{\square}
$$

The result was established through a proof that, although being proof-theoretical, was not completely direct as it used an auxiliary translation. It is not clear how

\footnotetext{
${ }^{11}$ See the self-contained survey by Wolter and Zakharyaschev (2014), whose notation we have followed and which includes an extension of the Blok-Esakia theorem to intuitionistic modal logics.
} 
the proof could be extended to intermediate logics, the first reason being the lack of standard Gentzen calculi for those logics. Calculi that extend traditional sequent calculi with the use of labels have however been developed in recent years by many authors, and they have proved successful especially for the proof-theoretic investigation of philosophical and non-classical logics (see the bibliography of Negri 2011 for references). Labelled sequent calculi for intermediate logics and their modal companions have been provided in Dyckhoff and Negri (2012) together with a constructive, direct, and uniform proof of soundness and faithfulness of the modal embedding.

Labelled systems for logics characterized by a Kripke-style semantics appear in several guises, as labelled tableaux, natural deduction, or sequent calculi. All these systems take advantage of the generality and uniformity of relational semantics and have the following features in common:

- The language is enriched by expressions of the form $x: A$ to denote truth of $A$ at world $x$ and $x R y$ for the accessibility relation between worlds.

- Introduction and elimination rules (or right and left rules in the case of sequent calculi) are determined by the possible-world explanation of logical constants by unfolding the inductive definition of truth at a world.

- Rules for extensions of basic systems are found by translating the properties of characteristic Kripke frames into rules for the accessibility relation.

In the labelled sequent calculi introduced in Negri (2005) these guiding ideas are implemented through a methodology of extension of cut- and contraction-free sequent calculi with rules. The extension is realised in a way that allows the structural properties of the calculus to be maintained. More specifically, the properties of the accessibility relation of characteristic Kripke frames for each logic are obtained by the method of "axioms as rules" (Negri and von Plato 1998).

The above features are common to all proof systems obtained through the internalization of Kripke semantics. As a concrete example, the rules for the basic modal logic $\mathbf{K}$ are obtained from the semantic explanation of modality

$$
x \Vdash \square A \Longleftrightarrow \text { for all } y, x \text { Ry implies } y \Vdash A
$$

that gives the rules

$$
\frac{y: A, x: \square A, x R y, \Gamma \rightarrow \Delta}{x: \square A, x R y, \Gamma \rightarrow \Delta} L \square \quad \frac{x R y, \Gamma \rightarrow \Delta, y: A}{\Gamma \rightarrow \Delta, x: \square A} R \square \text { (y fresh) }
$$

Similarly, the rules for implication for intuitionistic propositional logic are obtained from

$$
x \Vdash A \supset B \Longleftrightarrow \text { for all } y, x \leqslant y \text { and } y \Vdash A \text { implies } y \Vdash B
$$


that gives the rules

$$
\begin{aligned}
\frac{x: A \supset B, x \leqslant y, \Gamma \rightarrow \Delta, y: A \quad y: B, x: A \supset B, x \leqslant y, \Gamma \rightarrow \Delta}{x: A \supset B, x \leqslant y, \Gamma \rightarrow \Delta} L \supset \\
\frac{\frac{x \leqslant y, y: A, \Gamma \rightarrow \Delta, y: B}{\Gamma \rightarrow \Delta, x: A \supset B} R \supset \text { (y fresh) }}{}
\end{aligned}
$$

Here the relation $\leqslant$ is a preorder, i.e. reflexive and transitive, which are the properties of the accessibility relation for the modal logic $\mathbf{S 4}$. These properties are formulated in the form of sequent rules as

$$
\frac{x \leqslant x, \Gamma \rightarrow \Delta}{\Gamma \rightarrow \Delta} \operatorname{Ref} \quad \frac{x \leqslant z, x \leqslant y, y \leqslant z, \Gamma \rightarrow \Delta}{x \leqslant y, y \leqslant z, \Gamma \rightarrow \Delta} \text { Trans }
$$

In this way, complete sequent calculi G3I and G3S4 are obtained from a uniform methodology for intuitionistic logic and $\mathbf{S} \mathbf{4}$, respectively (see Dyckhoff and Negri, 2012, for the full table of rules). The calculi have the important feature of having all the structural rules admissible and all the rules invertible. In particular, faithfulness of the embedding of Int into $\mathbf{S} 4$ is obtained by a simple proof by induction on the height of derivations, in the same way as soundness, and the result (theorem 7.2 ibid.)

$$
\text { G3I } \vdash \Gamma \rightarrow \Delta \text { if and only if G3S4 } \vdash \Gamma^{\square} \rightarrow \Delta^{\square}
$$

is obtained in both directions through an explicit proof transformation, without any use of non-constructive principles.

The identification of intuitionistic implication with classical strict implication is clear at the level of semantic explanation and is reflected in the rules of the labelled calculus for intuitionistic logic. This can be recognized as the first reason for the simplicity of the proof of the embedding. The second reason is the way different logics are obtained from a "core" logical system by the addition of rules for the accessibility relation. In fact, the properties and the corresponding rules are the same for intermediate logics between intuitionistic and classical logic and between $\mathbf{S} 4$ and S5. Intermediate logics are obtained by adding suitable axioms to Int. We obtain analytic proof systems for a wide class of such logics by converting such axioms into frame properties of their Kripke semantics and by converting the frame properties into rules to be added to the sequent calculus. All the seven interpolable intermediate logics are obtained in this way, but there are infinitely many more.

For example, Gödel-Dummett logic is characterized by the axiom

$$
(A \supset B) \vee(B \supset A)
$$

This axiom corresponds to a linear accessibility relation

$$
\forall x \forall y(x \leqslant y \vee y \leqslant x)
$$


and becomes the rule

$$
\frac{x \leqslant y, \Gamma \rightarrow \Delta \quad y \leqslant x, \Gamma \rightarrow \Delta}{\Gamma \rightarrow \Delta} \operatorname{Lin}
$$

The addition of the rule to G3I defines a complete relational proof system for Gödel-Dummett logic. ${ }^{12}$ In a similar way, complete proof systems are obtained for all intermediate logics characterised by frames that satisfy first-order properties known as geometric implications (Dyckhoff and Negri 2012). ${ }^{13}$

The embedding result is extended to such logics in a straightforward way because their modal companions are characterized by the same frame properties, hence the same added rules. Given an extension G3I ${ }^{e x t}$ of G3I with rules for $\leqslant$, we denote by G3S4 $4^{\text {ext }}$ the corresponding extension of G3S4; we have

$$
\text { G3I }^{\text {ext }} \vdash \Gamma \rightarrow \Delta \text { if and only if G3S4 } 4^{\text {ext }} \vdash \Gamma^{\square} \rightarrow \Delta^{\square}
$$

The embedding result can be extended also in the direction of subintuitionistic logics, that is, logics obtained by giving up combinations of the following conditions of Kripke semantics for intuitionistic logics (where we indicate the generic accessibility relation with $R$ rather than $\leqslant$ because in the absence of reflexivity and transitivity the accessibility relation is not a preorder):

1. Monotonicity of forcing: If $x \Vdash A$ and $x R y$, then $y \Vdash A$

2. Reflexivity: $\forall x \cdot x R x$

3. Transitivity: $\forall x y z . x R y \wedge y R z \rightarrow x R z$

4. Falsum non datur: $\forall x . x \nVdash \perp$

Restall (1994) presents axiomatizations and Henkin-style completeness proofs for all the 12 subintuitionistic logics obtained by giving up combinations of the above conditions, with the proviso that whenever monotonicity is present, also transitivity is (recall that the extension of monotonicity of forcing from atomic to arbitrary formulas requires transitivity). Their algebraic semantics has been investigated by Celani and Jansana (2001).

We obtain complete relational sequent calculi $\mathbf{G}_{3} \mathbf{I}_{S}^{\text {rest }}$ for these logics by modifying the calculus G3I with $S$ consisting of one or more of the following items:

1. Replace the initial sequent $x R y, x: P, \Gamma \rightarrow \Delta, y: P$ by $x: P, \Gamma \rightarrow \Delta, x: P$.

\footnotetext{
${ }^{12}$ As discussed in Section 8 of Dyckhoff and Negri (2012), analyticity is guaranteed by the possibility of restricting the above rule to labels $x$ and $y$ found in its conclusion.

${ }^{13}$ Indeed, by the replacement of rules with systems of rules, the method can be further extended to frame classes expressed by generalized geometric implications, which are firstorder properties with an arbitrary number of quantifier alternations (cf. Negri 2016), and even to arbitrary first-order frame conditions with the method detailed in Dyckhoff and Negri (2015).
} 
2. Remove the rule of reflexivity.

3. Remove the rule of transitivity.

4. Remove $L \perp$, i.e., $x: \perp, \Gamma \rightarrow \Delta$, and replace it with $x: \perp, \Gamma \rightarrow \Delta, x: \perp$.

We shall indicate with $\mathbf{G 3 I}_{0}$ the system obtained by all four of the above modifications, namely G3I $\mathbf{I}_{1,2,3,4}^{\text {rest }}$ With the same modifications but the first on the modal side (where initial sequents already have the modified form) we obtain complete relational calculi G3S4 ${ }^{\text {rest }}$ for their modal companions. Observe that G3S4 $4_{2,3,4}^{\text {rest }}$ is just $\mathbf{G} 3 \mathbf{K}_{4}^{\text {rest }}$, and will be denoted with $\mathbf{G} 3 \mathbf{K}_{0}$.

The restriction of the embedding of Int into $\mathbf{S} 4$ to subintuitionistic logics is not as straightforward as its extension to intermediate logics. We start with G3I $\mathbf{I}_{0}$ and $\mathbf{G} \mathbf{3} \mathbf{K}_{0}$. Whereas soundness of the ${ }^{\square}$-translation is unproblematic, faithfulness is not: the case in which the translated formula has the form $\square P$ and is principal in the last rule of the derivation requires, if the rule is $R \square$, use of reflexivity of the accessibility relation, and, if the rule is $L \square$, use of monotonicity. However, by modifying the $(\cdot)^{\square}$ translation to the identity on atomic formulas, we obtain another translation, $(\cdot)^{t}$, for which these problematic cases are avoided, soundness continues to hold, and the other cases in the proof of faithfulness are shown as in the proof of the embedding of G3I into G3S4 because they stay within the respective restrictions. Clearly, soundness and faithfulness are maintained also in the presence of Ref, Trans and Ex falso. Monotonicity $x R y, x: P, \Gamma \rightarrow \Delta, y: P$ requires a special mention because it required also transitivity for monotonicity on arbitrary formulas, i.e. $x R y, x$ : $A, \Gamma \rightarrow \Delta, y: A$ to be derivable. The $t$-translation is inadequate to obtain soundness because $x R y, x: P, \Gamma \rightarrow \Delta, y: P$ is not derivable in G3S4 $4^{\text {rest }}$. By the requirements made to get faithfulness of the $\square$ translation we obtain:

Theorem 3.1. (a) Let $\mathbf{G 3 I}_{S}^{\text {rest }}$ be any non-monotonic restriction of $\mathbf{G} 3 \mathbf{I}$, i.e. $S$ is a subset of $\{1,2,3,4\}$ including 1 . Then $\mathbf{G 3 \mathbf { I } _ { S } ^ { r e s t }} \vdash \Gamma \rightarrow \Delta$ if and only if G3S4 $_{S}^{\text {rest }} \vdash \Gamma^{t} \rightarrow \Delta^{t}$.

(b) If $\mathbf{G} 3 \mathbf{I}_{4}^{\text {rest }} \vdash \Gamma \rightarrow \Delta$ if and only if $\mathbf{G} 3 \mathbf{S} \mathbf{4}_{4}^{\text {rest }} \vdash \Gamma^{\square} \rightarrow \Delta^{\square}$.

Proof. (a) Both soundness and faithfulness of the translation are proved by induction of the height of the derivation. The proof proceeds as the proof of theorem 7.2 of Dyckhoff and Negri (2012) but with a simplification owing to a reduction of the number of cases because of the identity translation of atomic formulas. In particular, there is no need of a separate lemma to account for the possibility of premisses, with conclusion in the range of the translation, that contains formulas not in the range of the translation.

(b) Follows as a special case from the proof of the above-mentioned theorem.

QED 
The result can be related to the algebraic results of Celani and Jansana (2005) that characterize weak Heyting algebras as strict implication fragments of normal modal algebras.

\section{Back to representations and applications to word problems}

The algebraic-topological representation theorems that arise from Stone representations constitute the backbone of the algebraic methods for philosophical logic that give the key ingredient in the traditional proofs of logical embedding results. Indeed, what can be regarded as the extensional part of representation results, i.e. the part concerned with the formalization of the notion of points of topological spaces, requires the use of non-constructive principles. On the other hand, we have seen that by the use of analytic proof theory we can establish the embedding results for intermediate and subintuitionistic logics into their modal companions in a direct, uniform, and constructive way. Indeed, we can further prove that logical (proof-theoretic) embeddings allow to recover the intensional (pointfree) part of representation results.

Suppose that we have a sound and faithful translation (embedding) from a logic $L_{1}$ to a logic $L_{2}$, that is, a map inductively defined on formulas

$$
*: L_{1} \longrightarrow L_{2}
$$

that satisfies

$$
\Gamma \vdash_{L_{1}} A \quad \text { iff } \quad \Gamma^{*} \vdash_{L_{2}} A^{*}
$$

Let $\mathcal{L}_{1}$ and $\mathcal{L}_{2}$ be the Lindenbaum algebras of $L_{1}$ and $L_{2}$, respectively, and define a map

$$
\begin{array}{rll}
f: \mathcal{L}_{1} & \rightarrow & \mathcal{L}_{2} \\
{[A]} & \mapsto & {\left[A^{*}\right]}
\end{array}
$$

Proposition 4.1. If the logics $L_{1}$ and $L_{2}$ satisfy detachment and the deduction theorem, the map $f$ is well defined and injective.

Proof. To show that $i$ is well defined, i.e. that its definition does not depend on the choice of the class representative, we have to show that if $A$ and $B$ belong to the same class in $\mathcal{L}_{1}$ then $\left[A^{*}\right]=\left[B^{*}\right]$. This is an immediate consequence of soundness of the translation: from $\vdash_{L_{1}} A \supset \subset B$ we get, through detachment and the deduction theorem, $\vdash_{L_{2}} A^{*} \supset \subset B^{*}$. Injectivity of $i$ follows in the same way from faithfulness.

QED

Let $*$ be the modal translation that we have considered in Section 3.2 and let $L_{2}$ be any modal logic extending $\mathbf{S} 4$. We can extend $*$ to the language of $L_{2}$ by 
adding to the inductive definition of $*$ the clause

$$
(\square A)^{*} \equiv A^{*}
$$

Next, we endow $\mathcal{L}_{2}$ with an operator $i$ defined by

$$
i[A] \equiv\left[A^{*}\right]
$$

We have:

Proposition 4.2. The operator $i$ satisfies the dual properties of the operator of closure algebras:

1. $1 \leq i(1)$

2. $i(a) \wedge i(b) \leq i(a \wedge b)$

3. $i(a) \leq a$

4. $i(a) \leq i(i(a))$

Proof. 1. Immediate from the derivability in $L_{2}$ of $\square \top^{14}$. 2. By definition of $i$ and the inductive clause that defines ${ }^{*}$ for conjunction. 3, 4. By definition of $i$ and, respectively, the facts (proved by induction on $A$ ) that $A^{*} \vdash_{L_{2}} A$ and $A^{*} \vdash_{L_{2}} A^{* *}$.

QED

We recall that the open elements of an algebra with an interior operator are the elements $a$ such that $a=i(a)$. In the special case of the Lindenbaum algebra $\mathcal{L}_{2}$, the open elements are the equivalence classes $[A]$ such that $[A]=i[A] \equiv\left[A^{*}\right]$, i.e. such that $\vdash_{L_{2}} A \supset \subset A^{*}$. It is almost immediate to verify that for all $A$ in the language of $L_{2}$ there is $A^{\prime}$ (obtained by removing all boxes) in the language of $L_{1}$ such that $A^{*}=A^{* *}$. Therefore the open elements are given by classes of formulas equivalent in $L_{2}$ to translated formulas.

When $*$ is the modal translation, the map $f$ is clearly a congruence with respect to the operations of the algebra and we have that $\mathcal{L}_{1}$ is isomorphic to the algebra of open elements of $\mathcal{L}_{2}$.

By the identification of free Heyting algebras with the Lindenbaum algebras of intuitionistic logic, the algebraic-topological reading of the Int to S4-embedding becomes:

Every freely generated Heyting algebra is isomorphic to the algebra of open elements of a closure algebra.

A similar reading can be applied, with the appropriate class of algebras substituted for Heyting and closure algebras, to each of the logical embedding results we have presented. Although the result is not new, the use of analytic calculi will allow a change of perspective: instead of using algebras to solve problems

\footnotetext{
${ }^{14}$ Observe that it is not necessary to assume a constant $T$ since we can use a definition such as $\perp \supset \perp$.
} 
in the logics, we use the logics, and more especifically their well behaved calculi, to solve problems in the algebras.

The representation of Heyting algebras as algebras of open elements of a closure algebra is replaced by the following embedding:

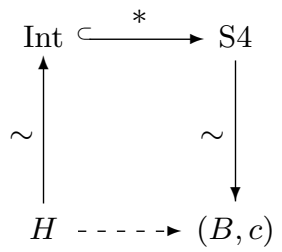

and more generally by

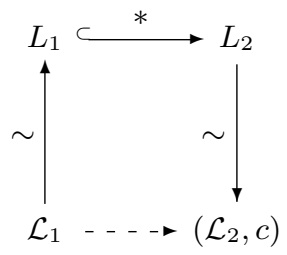

There is a more precise sense in which these embedding results can be interpreted in algebra, namely as reductions of classes of word problems: the question whether an inequality $a \leq b$ holds in a variety $\mathcal{L}_{1}$ of freely generated algebras/ordered structures can be reduced to the question whether the inequality $a^{*} \leq b^{*}$ holds in another variety $\mathcal{L}_{2}$. Soundness and faithfulness of the translation allow the transfer of both the problem and the solution between $\mathcal{L}_{1}$ and $\mathcal{L}_{2}$.

The general idea behind the use of sequent calculus in the solution of word problems in varieties of lattices is the following: first the defining properties of the lattice operations are translated into appropriate sequent calculus rules, for example, $a \wedge b \leq a$, and $c \leq a$ and $c \leq b$ imply $c \leq a \wedge b$, get translated to the rules (where $c \equiv a$ together with reflexivity gives the first axiom)

$$
\frac{a \leq c}{a \wedge b \leq c} \quad \frac{c \leq a \quad c \leq b}{c \leq a \wedge b}
$$

As a further step, the $\leq$ relation is substituted by the sequent arrow $\rightarrow$, and a sequent calculus is obtained. The sequent calculus can have a single formula both in the antecedent and succedent, or a multiset of formulas if distributivity properties also need to be encoded. Transitivity of the $\leq$ relation corresponds to the cut rule, and then it is shown that an atom of the form $a \leq b$ holds in the specific class of ordered structures if and only if the sequent is derivable in the sequent calculus. The calculus is formulated in such a way that it decomposes the problem of deciding whether $a \leq b$ holds by root-first application of the rules that pertains to the lattice operations in $a$ and $b$. Then cut elimination allows to solve the word problem because cut is the only rule that does not satisfy the subformula property. The elimination of transitivity is behind the solution of the 
word problem for free lattices in Whitman (1941), and the explicit translation of word problems into questions of cut elimination is at the basis of the work of Matsumoto (1965) for free lattices and of Tamura (1988) for ortholattices. The question as to what properties of a variety of algebras ensure cut elimination for the corresponding sequent calculus has been posed in precise terms and partially answered in Schulte Mönting (1981).

In the specific case of modal logic that we have considered here, the problem of deciding whether $a^{*} \leqslant b^{*}$ hols in a closure algebra is first translated to the question of whether the corresponding sequent $a^{*} \rightarrow b^{*}$ is derivable in $\mathbf{S} \mathbf{4}$ (or extension thereof). The next step is to use an analytic system to answer the question, as the labelled calculi presented in Dyckhoff and Negri $(2012,2016)$. Alternatively, one can use faithfulness of the translation to reduce the problem to a question of derivability in the corresponding intermediate logic. Again, the labelled calculus provides an analytic system that either produces a proof or a countermodel, by the methods of Negri (2014).

There is a more general sense in which proof theory can be employed for the solution of word problems in algebra, namely to answer the questions posed by uniform word problems, i.e. the question whether an atomic formula (of the form $a \leq b$ or $a=b$ ) follows from a finite number of atomic formulas $\Gamma$ used as assumptions, say $a_{1} \leq b_{1}, \ldots, a_{n} \leq b_{n}$. Thanks to the internalization of the derivability relation in sequent calculus, the problem can be expressed as the question of whether the sequent $\Gamma \rightarrow a \leq b$ is derivable. This problem is more general and the inequality cannot be translated as a sequent arrow. It can nevertheless be faced with sequent calculus by leaving the inequality between terms as atomic formulas in the logic and translating the axioms of the algebraic or ordered structure under consideration as rules that govern the behaviour of such relations, in the same way as transitivity or reflexivity were added as "nonlogical" sequent rules. The method has been employed to solve the uniform word problem for linear order (Negri, von Plato and Coquand, 2004), lattices (Negri and von Plato, 2002, 2004), linear lattices (Negri, 2005a, and by a different route in section 7.2 of Negri and von Plato, 2011), groupoids (Negri and von Plato, 2011), linear Heyting algebras (Dyckhoff and Negri, 2006), and ortholattices (Meinander, 2010).

\section{Concluding remarks and related work}

Stone representation theorems are considered one of the most important achievements in the unification of logic and topology; they show that "growth in mathematical knowledge often results when two or more distinct but related fields are unified by the hypothesis of a partial structural analogy, which allows for the combination of their resources in the solution and discovery of problems" (Grosholz 1985). The importance of this unification has been compared to the one realized by Descartes between algebra and Euclidean geometry through the creation of analytic geometry (Grosholz, ibid.). 
Mormann (2005) describes the representational program initiated by Stone as one of the "success stories" of $20^{\text {th }}$ century mathematics, in a line of conceptual development that sees Russell's theory of definite descriptions and Carnap's construction of qualities out of elementary experiences as forerunners of what was successfully carried out by Stone, and Stone's work as a precursor of the later conceptualization of pointfree topology. Mormann (2013) goes further in analyzing the way topology has been neglected in $20^{t h}$ century philosophy of science despite the "spectacular results" obtained by Stone, and conjectures that the cause of this neglect is to be found in a reductionist logical philosophy, with its emphasis on the logical foundations of mathematics.

The role of logic mediated through axiomatics in Stone's unification of algebra and topology is presented as central by Schlimm (2008): a common set of axioms is instrumental in the transfer of notions and methods between the domain of deductive systems and topology through the axiomatization of Boolean algebras.

In this work we have proposed a change of perspective by pushing the role of logic further through the use of analytic proof theory. We have made a distinction between the intensional and the extensional parts of the algebraic-topological representation theorems and analyzed how the shift from the intensional part to the extensional one requires the use of non-constructive principles. We have outlined two ways in which such non-constructive principles can be isolated or even avoided: the first is through the use of formal topology, the second is through the methods of analytic proof theory. Our focus is on the second method, for two reasons: On the one hand, embedding results are provable in a direct and constructive way, and on the other hand, they make it possible to recover the intensional part of the representation results through proof-theoretical embeddings. We have also indicated how embedding results can be interpreted in algebra as reductions of classes of word problems, a way for obtaining applications in algebra of the methods of analytic proof theory.

Finally, I thank the organizers of the conference "Intensionality in Mathematics", held in May 2013 in Lund, that gave the occasion to present this work, and gratefully acknowledge the insightful and constructive comments by the referees.

\section{References}

Bezhanishvili, G. (2014) Leo Esakia on Duality in Modal and Intuitionistic Logics. Springer, Dordrecht.

Bezhanishvili, G., N. Bezhanishvili, D. Gabelaia, and A. Kurz (2010) Bitopological duality for distributive lattices and Heyting algebras. Mathematical Structures in Computer Science, vol. 20, pp. 359-393.

Cederquist, J., T. Coquand, and S. Negri (1998) The Hahn-Banach theorem in type theory. In Twenty-Five Years of Constructive Type Theory, G. Sambin and J. Smith (eds), Oxford University Press, pp. 57-72. 
Cederquist, J. and S. Negri (1996) A constructive proof of the Heine-Borel covering theorem for formal reals. In Types for Proofs and Programs, S. Berardi and M. Coppo (eds), Lecture Notes in Computer Science 1158, pp. 62-75, Springer.

Celani, S. and R. Jansana (2001) A closer look at some subintuitionistic logics. Notre Dame Journal Formal Logic, vol. 42, 225-255.

Celani, S. and R. Jansana (2005) Bounded distributive lattices with strict implication. Mathematical Logic Quarterly vol.51, pp. 219-246.

Chagrov, A. and M. Zakharyaschev (1997) Modal Logic. Oxford Logic Guides, vol. 35. The Clarendon Press, New York.

Coquand, T., G. Sambin, J. Smith, and S. Valentini (2003) Inductively generated formal topologies. Annals of Pure and Applied Logic, vol. 124, pp. 71-106.

Davey, B. A. and H. Priestley (1990) Introduction to Lattices and Order. Cambridge University Press.

Dummett, M.A.E. and E.J. Lemmon (1959) Modal logics between S4 and S5, Zeitschrift für Mathematische Logik und Grundlagen der Mathematik vol. 5, pp. 250-264.

Dunn, M. and G. Hardegree (2001) Algebraic Methods in Philosophical Logic. Oxford University Press.

Dyckhoff, R. and S. Negri (2006) Decision methods for linearly ordered Heyting algebras. Archive for Mathematical Logic, vol. 45, pp. 411-422.

Dyckhoff, R. and S. Negri (2012) Proof analysis in intermediate logics. Archive for Mathematical Logic, vol.51, pp. 71-92.

Dyckhoff, R. and S. Negri (2015) Geometrization of first-order logic, The Bulletin of Symbolic Logic, vol. 21, pp. 123-163.

Dyckhoff, R. and S. Negri (2016) A cut-free sequent system for Grzegorczyk logic, with an application to the Gödel-McKinsey-Tarski embedding. Journal of Logic and Computation, vol. 26, pp. 169-187.

Fourman, M.P. and Grayson, R.J. (1982) Formal spaces. In A.S. Troelstra and D. van Dalen (eds) The L. E. J. Brouwer Centenary Symposium, North-Holland 107-122.

Gambino, N. and P. Schuster (2007) Spatiality for formal topologies. Mathematical Structures in Computer Science, vol. 17, pp. 65-80.

Grosholz, E. R. (1985) Two episodes in the unification of logic and topology. British Journal for the Philosophy of Science, vol.36, pp. 147-157.

Gödel, K. (1933) Eine Interpretation des intuitionistischen Aussagenkalküls. Ergebnisse eines mathematischen Kolloquiums vol.4, pp. 39-40. English tr. in Gödel's Collected Works vol. I, pp. 300-303, 1986.

Johnstone, P. (1982) Stone Spaces. Cambridge Studies in Advanced Mathematics, vol. 3, Cambridge University Press.

Martin-Löf, P. (1984) Intuitionistic Type Theory. Bibliopolis.

Matsumoto, K. (1965) Word problems for free lattices (in Japanese). Kiyo of Nara Technical College, vol.1, pp. 53-59. 
McKinsey, J.C.C. and A. Tarski (1944) The algebra of topology. Annals of Mathematics, vol. 45, pp. 141-191.

McKinsey, J.C., and A. Tarski (1948) Some theorems about the sentential calculi of Lewis and Heyting. Journal of Symbolic Logic, vol. 13, pp. 1-15.

Meinander, A. (2010) A solution of the uniform word problem for ortholattices. Mathematical Structures in Computer Science, vol. 20, pp. 625-638.

Mormann, T. (2005) Description, construction and representation. From Russell and Carnap to Stone. In G. Imaguire and B. Linsky (eds), On Denoting 1905 - 2005, Philosophia Verlag, München, pp. 333-360.

Mormann, T. (2013) Topology as an issue for history of philosophy of science. In H. Andersen et al. (eds), New Challenges to Philosophy of Sciences, Springer, pp. 423-434.

Negri, S. (1996) Stone bases, alias the constructive content of Stone representation. In A. Ursini and P. Aglianò (eds) Logic and Algebra, Lecture Notes in Pure and Applied Mathematics vol. 180, pp. 617-636, M. Dekker, New York.

Negri, S. (2002) Continuous domains as formal spaces. Mathematical Structures in Computer Science, vol. 12, pp. 19-52.

Negri, S. (2005) Proof analysis in modal logic. Journal Philosophical Logic, vol. 34, pp. 507-544.

Negri, S. (2005a) Permutability of rules for linear lattices. In C. Calude and H. Ishihara (eds) "Constructivity, Computability, and Logic" Journal of Universal Computer Science, vol.11, pp. 1986-1995.

Negri, S. (2011) Proof theory for modal logic, Philosophy Compass, vol. 6, pp. 523-538.

Negri, S. (2014) Proofs and countermodels in non-classical logics. Logica Universalis, vol. 8, pp. 25-60.

Negri, S. (2016) Proof analysis beyond geometric theories: from rule systems to systems of rules. Journal of Logic and Computation, vol. 26, pp. 513-537.

Negri, S. and J. von Plato (1998) Cut elimination in the presence of axioms, The Bulletin of Symbolic Logic, vol. 4, pp. 418-435.

Negri, S. and J. von Plato. Permutability of rules in lattice theory (2002) Algebra Universalis, vol. 48 (2002), pp. 473-477.

Negri, S. and J. von Plato (2004) Proof systems for lattice theory Mathematical Structures in Computer Science, vol. 14, pp. 507-526.

Negri, S. and J. von Plato (2011) Proof Analysis. Cambridge University Press.

Negri, S., J. von Plato, and T. Coquand (2004) Proof theoretical analysis of order relations. Archive for Mathematical Logic, vol. 43, pp. 297-309.

Negri, S. and D. Soravia (1999) The continuum as a formal space, Archive for Mathematical Logic, vol. 38, pp. 423-447.

Negri, S. and S. Valentini, S. (1997) Tychonoff's theorem in the framework of formal topologies. The Journal of Symbolic Logic, vol.62, pp. 1315-1332. 
Priestley, H.A. (1970) Representation of distributive lattices by means of ordered Stone spaces. Bulletin of the London Mathematical Society, vol. 2, pp.186-190.

Rasiowa, H. and R. Sikorski (1963) The Mathematics of Metamathematics. Monografie Matematyczne, Tom 41. Panstwowe Wydawnictwo Naukowe, Warsaw.

Restall, G. (1994) Subintuitionistic logics. Notre Dame Journal of Formal Logic, vol. 35, 116-129.

Sambin, G. (1987) Intuitionistic formal spaces - a first communication. In D. Skordev (ed) Mathematical Logic and its Applications, Plenum Press, pp. 187-204.

Schlimm, D. (2008) Bridging theories with axioms: Boole, Stone, and Tarski. In B. van Kerkhove (ed) New Perspectives on Mathematical Practices, World Scientific, pp. $222-235$.

Schulte Mönting, J. (1981) Cut elimination and word problems for varieties of lattices, Algebra Universalis, vol. 12, pp. 290-321.

Stone M.H. (1936) The theory of representations of Boolean algebras. Transactions of the American Mathematical Society, vol. 40, pp. 37-111.

Stone M.H. (1937) Topological representation of distributive lattices and Brouwerian logics. Casopis pro pěstování matematiky a fysiky, vol. 67, pp. 1-25.

Tamura, S. (1988) A Gentzen formulation without the cut rule for ortholattices. Kobe J. Math. vol. 5, pp. 133-150.

Troelstra, A. and H. Schwichtenberg (1996) Basic Proof Theory, Cambridge University Press, (2nd ed. 2000).

Whitman, P. (1941) Free lattices. Annals of Mathematics, vol. 42, pp. 325-330.

Wolter, F. and M. Zakharyaschev (2014) On the Blok-Esakia theorem. In G. Bezhanishvili (ed) op. cit., pp. 99-118. 